Cuadernos de Lingüística Hispánica N. ${ }^{\circ} 21$

ISSN 0121-053X

Enero-Junio 2013; pp. 55-70

\title{
Diálogo democrático y políticas lingüísticas: una propuesta*
}

\section{Democratic dialogue and linguistic policies: a proposal}

LILIAN PAOLA TORRENTE PATERNINA**

Iptorrentep@unal.edu.co

* Este artículo muestra resultados de una investigación expuesta en el XXVII Congreso Nacional y I Internacional de Lingüística, Literatura y Semiótica realizado en la Universidad Pedagógica y Tecnológica de Colombia, Tunja (9-12 de octubre de 2012).

*** Estudiante de la Maestría en Lingüística, Universidad Nacional de Colombia; Especialista en Pedagogía, Universidad Pedagógica Nacional, de Colombia; Licenciada en Lenguas Modernas, Universidad Surcolombiana; asesora pedagógica de la Escuela Virtual para América Latina y el Caribe del Programa de Naciones Unidas para el Desarrollo; docente ocasional del Departamento de Lenguas Extranjeras, Universidad Nacional de Colombia. 


\section{Resumen}

El diseño y formulación de las políticas públicas destinadas al uso y/o promoción de lenguas extranjeras o políticas lingüísticas públicas no contempla un ejercicio participativo de los estamentos de la sociedad que se verían beneficiados o afectados por la implementación de la misma, ya que se ha dejado en manos de los expertos en el tema la responsabilidad de elaborar ese tipo de políticas (como el Plan Nacional de Bilingüismo). El papel de los ciudadanos como participantes en la elaboración y/o evaluación de las políticas lingüísticas públicas para la promoción y enseñanza de lenguas extranjeras es prácticamente inexistente y el Estado colombiano no ha establecido unas estrategias de participación sobre el tema de políticas lingüísticas relacionadas con las lenguas extranjeras; por ello, sería interesante utilizar el Diálogo Democrático como una herramienta participativa para el diseño de una política aterrizada en el contexto, involucrando a todos los actores que puedan beneficiarse 0 verse afectados por la implementación de una política de este tipo.

Palabras clave: políticas públicas, políticas lingüísticas, promoción, lenguas extranjeras, participación ciudadana, diálogo democrático.

\section{Abstract}

The design and writing of public policies aimed at the use and/or promotion of foreign languages or public linguistic policies does not take into account a participatory effort from the different groups of society that will by benefitted or affected by their implementation; on the contrary, the responsibility of developing these policies (such as the Colombian National Bilinguism Plan) has been left in the hands of experts in the topic. The role of citizens as participants in the development and/or evaluation of public linguistic policies for the promotion and teaching of foreign languages is practically inexistent and the Colombian State has not established participation strategies. This paper proposes the use of democratic dialogue as a participatory tool for the design of more contextualized policies, that involve all the agents benefitted or affected by their implementation. The most appropriate way for the government and society to come together is through the exercise of those essential rights that allow and guarantee optimum conditions for dialogue, debate, criticism, dissent, and open discussion among parties.

Key words: public policies, linguistic policies, promotion, foreign languages, participatory citizenship, democratic dialogue. 


\section{Introducción}

El diseño de políticas públicas destinadas al uso y/o promoción de la lengua materna, lenguas extranjeras o lenguas nativas en Colombia ha seguido diferentes caminos: con respecto al uso de la lengua materna o las lenguas extranjeras, se recurre a los expertos (Academia de la Lengua, Instituto Caro y Cuervo, universidades, entes extranjeros -British Council, por ejemplo-) para el diseño de las mismas; caso contrario ocurre con las lenguas amerindias y/o nativas de grupos étnicos (afrodescendientes, raizales, indígenas), donde sí se ha contado con unos mecanismos de consulta para la creación e implementación de leyes y políticas (Ley de Lenguas Nativas) para su conservación y revitalización, aunque cabe resaltar que estas consultas o participación de estos grupos han sido cerrados y destinados sólo a estos grupos, y no están abiertas a la población general.

El caso particular al que me referiré es al de la creación y diseño de políticas públicas para la promoción del inglés como lengua extranjera en nuestro país, ya que como mencioné anteriormente, es de exclusiva atención de la comunidad experta que en este caso es el British Council. Como afirma Fischer (2009), [...] "given the dominance of expertise in the decision process [...] The institutions and practices of modern societies are anchored to professional knowledge, leaving decision makers highly dependent on expert judgments" (pp. 2-3). La difusión de estas políticas es posterior, cuando ha sido diseñada y aprobada por el gobierno y el Ministerio de Educación, y es simplemente informada a la ciudadanía en general, sin tenerla en cuenta, o a las múltiples entidades que estarían interesadas y que pudieran aportar al diseño de esta política, lo que ha sido muy criticado por diferentes estamentos como las universidades, al no ser llamadas a participar en este proceso, a pesar de que son sus facultades de educación o ciencias humanas quienes se encargan de formar a los docentes en lenguas extranjeras, quienes juegan un papel fundamental en el cumplimiento de las políticas establecidas.

El papel de los ciudadanos como participantes en la elaboración y/o evaluación de las políticas lingüísticas públicas para la promoción y enseñanza de lenguas extranjeras es 
prácticamente inexistente. Desafortunadamente, el Estado colombiano no ha establecido unas estrategias de participación sobre el tema de políticas lingüísticas relacionadas con las lenguas extranjeras; frente a esto y pese a entrar a competir en el mercado global y a las exigencias del fenómeno de la globalización, ha mantenido una posición pasiva y excluyente al momento de plantear una política bilingüe.

Frente a lo anterior vale la pena preguntarse enuestra visión de país debería incluir la meta de ser bilingïe en inglés? A nivel general, se afirma que es necesario contar con una población altamente competente en inglés para afrontar los desafíos de la globalización, pero la pregunta jamás se ha formulado a la población general: estudiantes, padres de familia, empleados, empresas, etc. En Colombia, la opinión general está de acuerdo con la promoción del inglés, pero no hay una motivación real, e incluso las políticas diseñadas (las cuales no tienen en cuenta el contexto monolingüe, ni las condiciones de escolarización) parecieran que no han tenido un efecto significativo en la población a la cual está destinada (estudiantes de todos los niveles educativos). De acuerdo con Sánchez Jabba (2012), el análisis de las pruebas Saber 11 de los años 2007 al 2010, presentadas por estudiantes colombianos de último grado de educación secundaria, mostró que el 2\% alcanzó el nivel B1 (intermedio) y menos del 1\% logró el nivel B2 (intermedio avanzado). Este es un indicador claro de que la política de bilingüismo no está dando los resultados esperados, teniendo en cuenta que son los colegios privados bilingües quienes obtienen los mejores puntajes en estas pruebas.

Ordoñez (2008) se pregunta cuál es el tipo de conocimiento que deberían poseer los tomadores de decisiones cuando se diseñan las propuestas enfocadas al desarrollo de habilidades comunicativas en el contexto de la escuela y de la mayoría de la población colombiana para que en realidad se pueda lograr una proficiencia bilingüe. Concluye que en el diseño de las políticas se deben tener en cuenta las variables del contexto para producir una política realista en nuestro contexto monolingüe. Para lograr esto, la participación ciudadana se vuelve una condición sine qua non para que se elabore una política lingüística contextualizada en Colombia, su aporte se vuelve un pilar de la política a desarrollar, en tanto que ella será quien refleje los resultados.

De manera similar, de Mejía, A. y Fonseca, L. (2006) reconocen que los actores que serían los directos beneficiarios de una política de bilingüismo son quienes deberían trabajar en equipo para consolidar una política de tipo inclusivo para desarrollar un sistema de educación bilingüe de excelencia en el país que reconozca el multilingüismo y el pluriculturalismo de la nación. 


\section{Política linguiística}

Existen varias aproximaciones a la definición de política lingüística, Siguan (2001) expone una definición del término:

A este carácter espontáneo de los comportamientos lingüísticos individuales y colectivos hay que añadir la existencia de individuos o de instituciones públicas que en determinados momentos y por distintas razones adoptan decisiones que pretenden influir sobre los comportamientos lingüísticos individuales y colectivos. Estas decisiones, los motivos que las mueven y los objetivos que persiguen constituyen lo que conocemos como política lingüística (p. 271).

Bergenholtz y Tarp (2005a) definen la política lingüística como "la regulación intencional de las relaciones interlingüísticas o intralingüísticas, o sea, de las relaciones entre las lenguas o dentro de una misma lengua [...]". Aclaran que la política interlingüística es de tipo prescriptivo porque es determinado por las autoridades públicas, y tienen como objetivo el favorecimiento o la imposición de una lengua -entre otros-. Shohamy (2009) citada por Alonso Cifuentes et al (2012) afirma que "existen mecanismos de política que crean prácticas de lenguaje, al imponer y negociar creencias sobre este. Dichos mecanismos incluyen reglas y regulaciones, exámenes de lenguaje, el lenguaje en el espacio público, mitos, propaganda y, en particular, políticas lingüísticas educativas" (p. 36).

Schmidt (2006) establece que las políticas lingüísticas tienen que ver con el desarrollo de políticas públicas, cuyo objetivo es utilizar la autoridad del Estado para afectar varios aspectos del estatus y el uso de las lenguas por las personas bajo la jurisdicción del Estado.

El punto común de estas definiciones es que la autoridad o el establishment es quien define las políticas lingüísticas a nivel de nación o estado, para que sean denominadas e identificadas como tal. Pero muchas veces, estas políticas perfectamente enunciadas y emanadas de las autoridades, tienen solamente un carácter discursivo, ya que no se llevan a la práctica por falta de voluntad política o por falta de una planificación linguística que Tollefson (2008) define como los esfuerzos deliberados para afectar la estructura, la función, y la adquisición de las lenguas, función que usualmente cumple (o incumple) el Estado, y la política linguística simplemente se queda en el carácter discursivo, sin ninguna aplicación práctica. Ricento (2006) explica que además de las políticas linguísticas y las prácticas, las "ideologías sobre la lengua en general y sobre idiomas específicos en particular tienen efectos reales sobre ellas" (p. 9). Aquí se refleja que a veces es más importante el uso de la 
lengua y las creencias que se tienen sobre ellas que la misma política implementada, lo que podría explicar el porqué muchas veces estas políticas no tienen resultados en la práctica. Aunque existe un obstáculo teórico, ya que hay una desarticulación entre las ciencias sociales con las ciencias de las políticas lingüísticas; varios autores, entre ellos Dua, 1996 y Schmidt, 2006 han empezado a establecer vínculos con las ciencias políticas definiendo los componentes que se pueden volver un continuo desde la lingüística hasta la ciencia política y viceversa. Siempre la política lingüística ha sido entendida como política educativa que decide una opción de escolarización en determinada lengua o lenguas, y no per se, donde existen unas implicaciones más profundas al hecho de que en una política de estado como el PNB se declare que el bilingüismo es la meta principal para el 2019.

\section{Plan Nacional de Bilinguiismo 2014-2019 y la participación ciudadana}

La base para la elaboración de la política del Plan Nacional de Bilingüismo fue la contratación del British Council para la ejecución de tres estudios diagnósticos, así: el primero midió la competencia comunicativa en inglés de 3.422 docentes de lengua extranjera; luego un estudio sobre el conocimiento pedagógico de enseñanza de lengua extranjera con 243 docentes usando el examen TKT y el tercer diagnóstico fue una alianza con el ICFES para la aplicación de una prueba a 2.467 estudiantes de colegios públicos y 1.293 de colegios privados (sin incluir colegios bilingües) (Usma, 2009). El Plan Nacional de Bilingüismo fue publicado en el boletín oficial del Ministerio de Educación Al tablero y posteriormente a través del decreto 3870 de 2006 del Ministerio de Educación Nacional, se adoptó el Marco Común Europeo de Referencia «como el sistema de referencia para los procesos de aprendizaje, enseñanza y evaluación adelantados en Colombia [en el área de idiomas]». Luego fue ratificado por el decreto 4904 de 2009, donde además se le otorga a los organismos de cooperación internacional la ventaja de no tener que acreditar sus programas de idiomas, a diferencia de los institutos para el trabajo y el desarrollo humano domiciliados en Colombia. ¿Cuál es el propósito de adoptar a rajatabla un enfoque de idiomas producido por el Consejo Europeo, donde se habla de plurilingüismo y multiculturalidad, que no tiene nada que ver en el contexto colombiano? El discurso pasado de moda de que Colombia es una nación multilingüe y multicultural se queda en los resguardos indígenas, ya que ellos son quienes hablan la lengua ancestral más el español -porque necesitan el idioma del mundo del "blanco"-, incluso a costa de su primera lengua, en peligro de extinción. El contexto colombiano que es mayoritariamente monolingüe en español exigiría un estudio más concienzudo de qué es lo que necesitamos para lograr esa política de estado de bilingüismo, que además deja por fuera las otras lenguas extranjeras (francés, alemán, mandarín, etc.), ya que el énfasis está solamente en el inglés. 
No hubo ningún esfuerzo por parte del Ministerio de Educación en hacer un ejercicio participativo real para establecer una política consensuada sobre la promoción de una lengua extranjera en el territorio nacional. De acuerdo con el estudio que realizaron Velásquez y González (2003), en Colombia, a pesar de que existe mucha normatividad para que se dé una participación activa por parte de los ciudadanos, "esa riqueza [de normas] no parece traducirse suficientemente en la movilización ciudadana en torno a lo público, en la democratización de la gestión y, sobre todo, en la incidencia de la ciudadanía en la formulación de las políticas públicas" (p. 26). El problema no es solamente de los ciudadanos, sino también del aparato estatal que utiliza los mecanismos de participación ciudadana como dispositivos para justificar las políticas que ya han formulado. En el caso del Ministerio de Educación, la única participación que tuvieron los actores fueron los diagnósticos a los docentes y a estudiantes, pero nunca se consultó a las universidades, los padres de familia, las empresas u otros estamentos de la sociedad para buscar una política más inclusiva. La "democratización" se ve desde la perspectiva del acceso de un conocimiento -tener acceso a una formación en lenguas extranjeras que usualmente es de la élite- a la población menos favorecida.

Lo anterior, devela el desconocimiento y el yerro del Estado en su manera de plantear sus políticas, como el de hacer su política. En el hecho de que desconoce los procesos de diálogo, la deliberación y la negociación como herramientas de participación válidas y necesarias en una sociedad donde uno de los retos de la globalización es el de afianzar los procesos democráticos. Por ello, sería interesante utilizar el Diálogo Democrático como una herramienta participativa para el diseño de una política aterrizada en el contexto, involucrando a todos los actores que puedan beneficiarse o verse afectados por la implementación de una política de este tipo. La manera idónea en que el gobierno y la sociedad se compenetren es mediante el ejercicio de aquellos derechos que permiten y garantizan las condiciones óptimas del diálogo, el debate, la crítica, el disenso y la discusión abierta entre estas dos entidades; es decir, el ejercicio del diálogo entre estos actores permite conocer, diagnosticar la problemática presente y futura y plantear soluciones concienzudas, tanto mediatas como a largo plazo acordes con la necesidad planteada, como lo expresa Amartya Sen (2000).

Mediante el diálogo se abren canales con los cuales se construye confianza y entendimiento, sorteando así las diferencias y generando resultados eficaces y eficientes a través de la conversación o negociación ya sea a nivel comunitario, social, administrativo o estatal. 


\section{Diálogo Democrático}

El Diálogo Democrático es una expresión que se utiliza, sobre todo, en América Latina y el Caribe para el reforzamiento de la gobernabilidad democrática (Pruitt y Thomas, 2008). Se puede definir como "el proceso en el que las personas se reúnen para construir confianza y entendimiento muto más allá de las diferencias y para generar resultados positivos a través de la conversación" (p. 9). Una ampliación de esta definición la proporciona IDEA Internacional (1999, citado por Pruitt y Thomas, 2008), así "el diálogo se diferencia del debate en que el primero promueve la diversidad de pensamientos y opiniones, en lugar de suprimirlos [...] En la práctica del diálogo, se acuerda que las ideas o creencias de una persona no tienen prioridad sobre las de otras" (p. 21).

El diálogo democrático (caracterización que recibe el diálogo que pretende sortear problemas de relevancia social y estatal dentro del marco internacional y que pretende reforzar la gobernabilidad democrática), debe enfrentar los siguientes desafíos, a saber:

3.1. Ocuparse de la complejidad de la problemática, ya sea por su carácter mutable que aborde en todo su espectro y ámbito.

3.2. Coordinar el significado, lo cual implica al tiempo coordinar el entendimiento de las diferentes posturas en relación con el tema objeto de diálogo a fin de lograr un criterio unificado.

3.3. Producir innovación, es decir, que no se caiga en la vieja costumbre, sino al contrario, que se le cuestione, se le desafíe y se proyecten cambios substanciales en todos los niveles.

3.4. Posibilitar la deliberación que ofrezca a su vez alternativas que permitan la toma de decisiones que implican para uno u otro actor concesiones en relación con sus posturas iniciales.

3.5. Producir resultados sostenibles. Pese a que en ocasiones por la situación problema se requieren medidas mediatas, estas suelen ser de carácter temporal y efímero. Se debe procurar soluciones que mantengan una perspectiva a largo plazo, donde se vincule tanto a los interesados como a los beneficiarios. (Pruitt y Thomas, 2008, pp. 15-16).

Existen tres criterios, según Pruitt y Thomas (2008), que ayudan a diferenciar los diálogos democráticos: 
- Objetivo: abordar problemas sociales complejos que las instituciones existentes no están atendiendo de forma adecuada.

- Participantes: un microcosmos del sistema que crea el problema y que debe formar parte de la solución.

- Proceso: un diálogo abierto e incluyente, que permite la construcción de la confianza necesaria para alcanzar acuerdos sobre acciones concretas (p. 25).

Para resumir, el siguiente cuadro expone un sumario sobre el enfoque dialógico, sus principios, objetivos, cualidades y conductas.

\begin{tabular}{|c|c|c|c|}
\hline \multicolumn{4}{|c|}{ El enfoque dialógico } \\
\hline $\begin{array}{l}\text { Principios } \\
\text { rectores }\end{array}$ & Objetivos & Cualidades & Conductas \\
\hline $\begin{array}{l}\text { - Inclusividad } \\
\text { - Apropiación } \\
\text { compartida } \\
\text { - Aprendizaje } \\
\text { - Humanidad } \\
\text { - Perspectiva de } \\
\text { largo plazo }\end{array}$ & $\begin{array}{l}\text { - Lograr el compromiso de } \\
\text { participación de todas las partes del } \\
\text { sistema } \\
\text { - Crear condiciones para el cambio } \\
\text { sobre la base de los temas que } \\
\text { importan } \\
\text { - Promover el aprendizaje yfacilitar una } \\
\text { comprensión más profunda } \\
\text { - Crear la sensación de seguridad } \\
\text { necesaria para lograr apertura en los } \\
\text { participantes } \\
\text { - Promover el compromiso para con } \\
\text { el logro de cambios sostenibles }\end{array}$ & $\begin{array}{l}\text { - Respeto } \\
\text { - Transparencia } \\
\text { - Apertura } \\
\text { - Empatía } \\
\text { - Autenticidad } \\
\text { - Paciencia } \\
\text { - Flexibilidad }\end{array}$ & $\begin{array}{l}\text { - Indagar para aprender } \\
\text { - Compartir los conocimientos } \\
\text { - Escuchar con empatía } \\
\text { - Ofrecer comentarios sobre lo que se ha } \\
\text { escuchado } \\
\text { - Explorar los supuestos subyacentes, tanto } \\
\text { propios como de los demás } \\
\text { - Reconocer las emociones, tanto como las } \\
\text { ideas y las opiniones } \\
\text { - Modificar el rumbo de manera que se } \\
\text { reflejen los nuevos conocimientos o la } \\
\text { nueva comprensión }\end{array}$ \\
\hline
\end{tabular}

Fuente: Pruitt, B. y Thomas, P. (2008) Diálogo Democrático: Un manual para practicantes (p. 49).

\section{Propuesta de diseño de una política pública (linguiística) para la promoción y el uso de una lengua extranjera (inglés)}

Siguiendo el protocolo de Diálogo Democrático: Un Manual para Practicantes, se plantea la siguiente guía para el diseño de un proceso de diálogo democrático: 
Guía. Diseño del proceso. En: Pruitt, B. y Thomas, P. (2008) Diálogo Democrático:

Un manual para practicantes (p. 86).

Proceso de diálogo que se está diseñando

Contexto

¿Cuál es el contexto general en el que este proceso tendrá lugar?

Premisas subyacentes

Exponga en forma explícita y simple las razones por las que se considera importante y estratégico realizar tal proceso dentro del contexto especificado. ¿Qué supuestos tenemos?

Cambios que se buscan

Exponga en forma explícita los objetivos o cambios deseados que este proceso busca generar.

Relación con otros procesos

Identifique cualquier relación que existe (o necesite crearse) entre esta iniciativa y otras iniciativas o procesos relacionados con los cambios deseados.

Objetivos específicos de este proceso

Exponga en forma explícita los objetivos específicos o cambios deseados del proceso que se está diseñando. Expréselos en términos de resultados inmediatos visibles, así como también de su impacto deseado con el paso del tiempo.

Indicadores de éxito

- Resultado inmediato: ¿Cuáles serán los indicadores inmediatos de éxito?

- Impacto con el paso del tiempo: ¿Cómo se verá ese éxito a futuro?

Condiciones mínimas requeridas

Identifique y exponga en forma explícita ciertas condiciones de las cuales depende el éxito de este proceso. A menudo las damos por sentado, por lo tanto, la idea es explicarlas.

Análisis de las fuerzas de los factores del entorno que merezcan consideración

Los procesos de diálogo nunca ocurren en el vacío. Las fuerzas externas (cosas que no controlamos) pueden favorecer o perjudicar el proceso.

\begin{tabular}{|c|c|c|c|}
\hline \multicolumn{2}{|c|}{ Fuerzas de apoyo } & \multicolumn{2}{|c|}{ Fuerzas de oposición } \\
\hline \multicolumn{2}{|c|}{ Factores que favorecen nuestro resultado deseado } & \multicolumn{2}{|c|}{ Factores que perjudican nuestro resultado deseado } \\
\hline \multicolumn{2}{|c|}{ 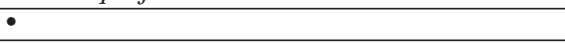 } & \multicolumn{2}{|c|}{$\cdot$} \\
\hline \multicolumn{2}{|l|}{$\bullet$} & \multicolumn{2}{|l|}{ - } \\
\hline \multicolumn{2}{|l|}{$\bullet$} & \multicolumn{2}{|l|}{$\bullet$} \\
\hline \multicolumn{4}{|c|}{$\begin{array}{l}\text { - Descripción con explicación de la lógica subyacente } \\
\text { - Formas de aprovechar las fuerzas de apoyo y de enfrentar las fuerzas de oposición } \\
\text { - Recursos necesarios (humanos, materiales) } \\
\text { - Matriz de acción: }\end{array}$} \\
\hline Tipo de acción & Meta de la acción & Duración & Quién \\
\hline \multicolumn{4}{|l|}{1.} \\
\hline \multicolumn{4}{|l|}{2.} \\
\hline \multicolumn{4}{|l|}{ c } \\
\hline Sostenibilidad & & & \\
\hline
\end{tabular}

Fuente: Creado por Philip Thomas para OAS/PROPAZ (1997, p. 86). 
¿Qué pasos ayudarán a asegurar la sostenibilidad de los impactos del proceso de diálogo?

En este orden de ideas y siguiendo la anterior guía proporcionada, mi propuesta para establecer un diálogo democrático para el diseño de una política pública (lingüística) orientada a la promoción de una lengua extranjera es la siguiente:

\subsection{Contexto}

Los estamentos de la sociedad no son incluidos en el proceso de elaboración de políticas públicas concernientes al uso y promoción de lenguas extranjeras. El gobierno recurre a entidades expertas para el diseño de éstas.

\subsection{Premisas subyacentes}

La población general es quien hace uso de la lengua sobre la que muchas veces se legisla, pero en donde la sociedad no tiene voz ni voto cuando se crean leyes, políticas o estrategias para el uso/promoción de otras diferentes a la lengua materna. La participación de la sociedad es fundamental para que se le dé un contexto genuino a una política de promoción de una lengua extranjera.

\subsection{Cambios que se buscan}

Al incluir la participación de la sociedad, del ciudadano común en la creación de una política linguística, se tiene en cuenta la visión de país que ellos puedan tener, comparada con las exigencias del tiempo histórico en el que nos encontramos, y con la visión de país que pueda tener el Estado.

\subsection{Relación con otros procesos}

El diseño de este tipo de políticas con involucramiento de todos los estamentos sociales se relacionaría principalmente con actores en educación, cultura, presupuesto, comercio, empresas.

\subsection{Objetivo específico de este proceso}

Una política más participativa, más contextualizada en el entorno. 


\section{Indicadores de éxito}

- Resultado inmediato: convocatoria para dialogar sobre las políticas públicas (lingüísticas) existentes, modificación de las mismas, participación activa de actores usualmente excluidos (población beneficiaria principal: estudiantes).

- Impacto con el paso del tiempo: legislación basada en las conclusiones del diálogo. Futuros ejercicios de diálogo para evaluar la implementación de estas políticas ya modificadas.

\subsection{Condiciones mínimas requeridas}

Definición de actores clave. Convocatoria amplia a los interesados en participar. Conocimiento sobre las políticas públicas (lingüísticas) actuales y análisis de las mismas por parte de los participantes. Propuestas aterrizadas en lo local, regional, nacional e internacional.

\subsection{Análisis de las fuerzas de los factores del entorno que merezcan consideración}

\begin{tabular}{|l|l|}
\hline \multicolumn{1}{|c|}{ Fuerzas de apoyo } & \multicolumn{1}{|c|}{ Fuerzas de oposición } \\
\hline - Estudiantes de todos los niveles académicos & - Entes internacionales con intereses \\
- Padres de familia & económicos \\
- Colegios, Instituciones de Formación para & - Ministerio de Educación \\
el Trabajo, Universidades & \\
- Empresas & \\
- Académicos & \\
\hline
\end{tabular}

\subsection{Estrategia}

- Utilización de redes sociales y tradicionales para la difusión de la convocatoria

- Diseño de una propuesta base sobre la cual se establece el diálogo democrático

- Matriz de acción: 
Cuadernos de Lingüística Hispánica No. 21

\begin{tabular}{|l|l|l|l|}
\hline \multicolumn{1}{|c|}{ Tipo de acción } & \multicolumn{1}{|c|}{ Meta de la acción } & \multicolumn{1}{c|}{ Duración } & \multicolumn{1}{c|}{ Quién } \\
\hline 1. Convocatoria & $\begin{array}{l}\text { Convocar a los actores } \\
\text { interesados }\end{array}$ & meses & Organizador \\
\hline 2. Diagnóstico & $\begin{array}{l}\text { Realizar un diagnóstico sobre } \\
\text { la temática para establecer } \\
\text { los puntos focales de interés }\end{array}$ & $\begin{array}{l}\text { Organizador } \\
\text { Equipo }\end{array}$ \\
\hline $\begin{array}{l}\text { 2. Diseño de propuesta } \\
\text { base }\end{array}$ & $\begin{array}{l}\text { Con base en el diagnóstico, } \\
\text { elaborar una propuesta base } \\
\text { para fomentar el diálogo sobre } \\
\text { los puntos focales de interés }\end{array}$ & $\mathbf{3 m e s e s}$ & $\begin{array}{l}\text { Organizador } \\
\text { Equipo }\end{array}$ \\
\hline 3. Evento & $\begin{array}{l}\text { Establecer la logística del evento } \\
\text { (actores, temas, sitios, difusión, etc.) }\end{array}$ & 1 mes & $\begin{array}{l}\text { Organizador } \\
\text { Equipo }\end{array}$ \\
\hline
\end{tabular}

\subsection{Sostenibilidad}

Realizar un seguimiento a los resultados del diálogo y su implementación a nivel de política pública.

\section{Conclusiones}

La selección del idioma que se va a enseñar en la educación pública y/o privada sumado a la ventaja del inglés como lengua hegemónica, hace que los procesos de políticas linguísticas obligatoriamente deban tener una importante participación de la ciudadanía, porque se está poniendo en juego la identidad de un país y de la posibilidad de desplazar la lengua materna por otra más "rentable". La presente es simplemente una propuesta de alternativa para utilizar un mecanismo de participación como lo es el Diálogo Democrático, que permita llegar a un consenso real sobre las políticas lingüísticas públicas, en donde todos los estamentos de la sociedad puedan tener voz y voto cuando se diseñan y se implementan. 


\section{Referencias bibliográficas}

Alonso Cifuentes, J., Casasbuenas, P., Gallo, B. y Torres, G. (2012). Bilingüismo en Santiago de Cali: Análisis de los resultados de las Pruebas SABER 11 y SABER PRO. Santiago de Cali: Universidad Icesi, pp. 36-40. Recuperado de http://www.icesi.edu.co/ biblioteca_digital/bitstream/10906/65354/1/bilinguismo_santiagocali_pruebas.pdf

Bergenholtz, H. y Tarp, S. (2005a). Política lingüística: Conceptos y deûniciones. En: 5th Symposium on Translation, Terminology and Interpretation in Cuba and Canada. Canadian Translators, Terminologists and Interpreters Council (eds). Recuperado de http:// www.cttic.org./e_publications.htm

Canale, G. (2011). Planificación y políticas lingüísticas en la enseñanza de lenguas extranjeras: el acceso al inglés en la educación pública uruguaya. Políticas Lingüísticas, 3(3), 45-74.

De Mejía, A. y Fonseca, L. (2006). Lineamientos para políticas bilingües y multilingües nacionales en contextos educativos lingüísticos mayoritarios en Colombia. Bogotá: Centro de Investigación y Formación en Educación Universidad de los Andes.

Delgado, E. (2004). Hacia una nueva articulación de los espacios lingüísticos y culturales. En: Pensar Iberoamérica, 6, mayo-agosto. Recuperado de http://www.oei.es/ pensariberoamerica/ric06a $04 . h \mathrm{tm}$

Dua, H. (1996). The politics of language conflict: Implications for language planning and political theory', Language Problems and Language Planning, 20, 1-17.

Fischer, F. (2009). Democracy and expertise: reorienting policy inquiry. Oxford: Oxford University Press.

Lagnado, J. (1999). The politics of language in Colombia and beyond. Ikala, 4, (7-8), 7-13.

Ministerio de Educación Nacional. (2005). Bases para una nación bilingüe y competitiva. $A l$ tablero, 37, octubre-diciembre. Recuperado de http://www.mineducacion.gov.co/1621/ propertyvalue-32266.html

Ordoñez, C. (2008). Education for bilingualism in international languages in a monolingual socio-linguistic context. Lenguaje, 36 (2), 353-384. 
Pruitt, B. y Thomas, P. (2008). Diálogo Democrático: Un manual para practicantes. Secretaría General de la Organización de los Estados Americanos, Washington; Instituto Internacional para la Democracia y la Asistencia Electoral, Estocolmo; Programa de las Naciones Unidas para el Desarrollo, Nueva York. Recuperado de http:// www.democraticdialoguenetwork.org/index.pl?lang=es

Ricento, T. (2006). An Introduction to Language Policy: Theory and Method. Malden: Blackwell Publishing.

Rodríguez Molano, M. (2009). Entrevista. Reflexiones sobre el bilingüismo en Colombia. $E l$ Educador, Noviembre. Recuperado de http://www.eleducador.com/images/stories/ documentos_descarga/Revistas_eleducador/5588_Entrevista.pdf

Saavedra, A. (2004). Los colombianos en Estados Unidos y la creación de una comunidad transnacional: por una nueva política de la lengua en Colombia. Colombia Internacional, 59, enero-junio, pp. 74-103.

Sánchez Jabba, A. (2012). El bilingüismo en los bachilleres colombianos. Documentos de trabajo sobre economía regional. Cartagena: Centro de Estudios Económicos Regionales del Banco de la República.

Schmidt, R. (2006). Political Theory and Language Policy. En: Ricento, T. (ed) An Introduction to Language Policy: Theory and Method. Malden: Blackwell Publishing, pp. 95-110.

Schiffman, H. (1996). Linguistic Culture and Language Policy. London: Routledge.

Sen, A. (2000). Desarrollo y libertad. Ciudad de México: Planeta.

Shohamy, E. (2009). Language Teachers as Partners in Crafting Educational Language Policies? Ikala, revista de lenguaje y cultura, 14(22):22.

Siguan M. (2001). Bilingüismo y Lenguas en Contacto. Madrid: Alianza.

Spolsky, B. (2004). Language Policy. Cambridge: Cambridge University Press.

Tollefson, J. (2008). Language planning in education. En: Hornberger, N. (ed) Encyclopedia of language and education, $2^{\text {nd }}$ edition, Volume 1: Language policy and political issues in Education. Nueva York, Springer. 
Torres, R. (2007). Incidir en la educación. Polis, 5(16).

Usma Wilches, J. (2009). Education and Language Policy in Colombia: Exploring Processes of Inclusion, Exclusion, and Stratification in Times of Global Reform. Profile, 11, eneroabril.

Velásquez, F. y González, E. (2003). ¿Qué ha pasado con la participación ciudadana en Colombia? Bogotá, Fundación Corona. 